\title{
Enfoque integral de la salud durante la pandemia
}

María Elena Critto $^{1}$

\author{
${ }^{1}$ Facultad de Ciencias Médicas \\ Pontificia Universidad Católica \\ Argentina.
}

Fecha de recepción: 19-10-2020

Fecha de aceptación: 21-10-2020

Citar como: Critto ME. Enfoque integral de la salud durante la pandemia. CASUS. 2020;5(2):48-49

DOI: $10.35626 /$ casus.2.2020.281
Según los datos de la Organización Mundial de la Salud, 41 millones de personas mueren cada año por las enfermedades no transmisibles (ENT), lo que equivale aproximadamente al $71 \%$ de las muertes que se producen en el mundo (1), y al $77 \%$ de las muertes en nuestro continente (2). Las principales causas de mortalidad informadas son las cardiovasculares, cáncer, respiratorias y diabetes.

Las enfermedades crónicas no transmisibles afectan la calidad de vida de las personas, y se les atribuye a ellas una alta carga de enfermedad (69\% para nuestro continente) (2). En gran medida son reducibles y hasta evitables, disminuyendo por un lado los principales factores de riesgo modificables (alimentación inadecuada, inactividad física, consumo de tabaco y alcohol), promoviendo conductas y entornos saludables (alimentación saludable, actividad física, ambientes libres de humo, límites al consumo de sodio y de alcohol) (1); y por otro, facilitando el acceso a la salud para la detección temprana, control, seguimiento y tratamiento oportuno de las mismas. Sin embargo, durante la pandemia COVID-19 se observan obstáculos para el acceso a la salud y prevención. Se detallan a continuación algunos ejemplos:

a) Inequidad en el acceso a la salud: atención de la salud solo para pacientes con covid en detrimento de la atención a otras enfermedades; postergación y cancelación de los controles y consultas médicas por parte de la población por temor y hasta pánico al contagio; reducción significativa de estudios para la detección, diagnóstico y tratamiento de patologías no asintomáticas como tumores. Con el aislamiento obligatorio disminuyeron por ejemplo los estudios de diagnóstico cardiológicos, mamografías, endoscopias, análisis de sangre, control de la presión arterial, del sobrepeso, entre otros; dificultades en el acceso a controles médicos periódicos de las embarazadas y de la primera infancia; postergación en el calendario de vacunas de los niños, niñas y adolescentes; deficiencias en la contención social y en el cuidado de la salud mental y emocional en situaciones de enfermedad; políticas de avance y retroceso respecto a la salud que acarrean en una pérdida de credibilidad de la población en el sistema de salud; falta de claridad en la información sobre la salud, confusión y falsas noticias. 
El costo de estas asimetrías y demoras en el acceso a la salud, a los diagnósticos precoces, a los controles y tratamientos médicos, no solo se traducen en tratamientos tardíos y por ende más caros, sino también en una mayor carga de enfermedad, mayor posibilidad de secuelas, mayor pérdida de productividad, y probablemente en un aumento de la mortalidad por enfermedades que no recibieron diagnóstico y tratamiento tempranamente (3).

Cabe destacar como afecta a la salud no solo la situación de la pandemia, sino también la existencia de otras afecciones que perjudican la calidad de vida independientemente del COVID19, las cuales cuanto más precozmente se detecten, mayor probabilidad de éxito y disminución de la carga de enfermedad tienen.

La prevención es la mejor forma de abordaje de la problemática de la salud también en tiempos de pandemia. Para ello, es imprescindible un enfoque integral y de concientización de todos los sectores de la sociedad, teniendo en cuenta los múltiples factores que afectan la salud (4). En este sentido, las necesidades de la población exhortan a continuar estimulando la promoción de la salud en todas sus dimensiones en pos de la reducción de los factores de riesgo modificables (hábitos y comportamientos), y, de la mejora de los múltiples indicadores contextuales que asimismo afectan a la salud. Tales como las condiciones sociales, ambientales, educativas, laborales, y sanitarias, que, interrelacionadas entre sí, interactúan con la salud de la población, aumentando o disminuyendo la susceptibilidad a las enfermedades (5).

Campañas de concientización social, comunicación de datos confiables de salud de manera clara contribuyen a decisiones informadas. La transparencia en la información aumenta la confianza en los servicios de salud, y esta mayor confianza aumenta los controles médicos. Mejores decisiones a nivel individual y a nivel comunitario previenen enfermedades, reducen factores de riesgo, aumentan factores protectores y mejoran la calidad de vida.

Es indispensable alertar a toda la sociedad y a los sistemas de salud para no demorar durante la pandemia la cobertura y el acceso a la salud para la detección, prevención, control, seguimiento y tratamiento de las enfermedades como las no transmisibles que no desaparecen por la pandemia. Todas las personas tienen derecho al acceso a la salud en la medida de sus necesidades y todas las enfermedades merecen ser atendidas en tiempo y forma. En este sentido es esencial encontrar un equilibro donde se prioricen también las tareas de prevención, las que no solo permiten disminuir la mortalidad, sino también mejorar las probabilidades de cura y la calidad de vida de las personas.

\section{REFERENCIAS BIBLIOGRÁFICAS}

1. World Health Organization. 3. Organización Panamericana de la Enfermedades no transmisibles. 1 de junio de 2018. Datos y cifras. Acceso en octubre 2020. Disponible

en:https://www.who.int/es/newsroom/fact-

sheets/detail/noncommunicablediseases

2. World Health Organization. The Global Burden of Disease: 2004 update. Ginebra 2008. Acceso en octubre 2020. Disponible en: https://www.who.int/healthinfo/gl obal_burden_disease/2004_report_ update/en/
Salud. Factores de riesgo de las enfermedades no transmisibles en la Región de las Américas: Consideraciones para fortalecer la capacidad regulatoria. 2015. Acceso en octubre 2020. Disponible en: https://iris.paho.org/bitstream/hand le/10665.2/28227/9789275318669 _spa.pdf?sequence $=6 \&$ isAllowed $=$ $\mathrm{y}$

- Organización Panamericana de la Salud. Módulo de Principios de Epidemiología para el Control de Enfermedades (MOPECE) Segunda Edición Revisada.
Control de enfermedades en la población. 2011. Acceso en octubre 2020. Disponible en: https://www.paho.org/bra/index.ph p?option=com_docman \& view $=$ do wnload\&category_slug=informaca o-e-analise-saude096\&alias $=1274-$ modulosprincipios-epidemiologia-paracontrol-enfermedades-mopeceunidad-6-control-enfermedadespoblacion- $4 \&$ Itemid $=965$
Alan Davidson. Social Determinants of Health. A Comparative Approach. Oxford University Press. 2015. 